\title{
Evaluation of some new somaclones of sugarcane for yield and quality
}

\author{
Abo-Elwafa A. ${ }^{1}$, B.R. Bakheit ${ }^{1}$, A.B. El-Taib ${ }^{2 *}$ and N.Y. Noby ${ }^{2}$ \\ ${ }^{1}$ Department of Agronomy, Faculty of Agriculture, Assiut University, Egypt \\ ${ }^{2}$ Department of Agronomy, Faculty of Agriculture and Natural Resources. Aswan University, Egypt
}

\begin{abstract}
The study aims to determine the genetic variability among eleven somaclones of sugarcane obtained via immature leaves of the Egyptian commercial variety GT-54 9 at Agronomy Department, Faculty of Agriculture, Assiut University. Significant differences were found among the somaclones and also their donor, revealing to abundance genetic differences among them. The highest values of GCV and PCV were estimated in agronomic traits for cane yield (18.11 and 18.53\%) and in technological traits for sugar yield (17.65 and $17.76 \%)$ over two ratoon crops, respectively. The heritability in agronomic traits ranged from 50.39 (stalk diameter) to $98.46 \%$ (cane yield) and in technological traits varied from 73.02 (purity) to $98.78 \%$ (sugar yield) over the both ratoon crops. The obtained significant differences coupled with GCV, PCV and heritability estimates explained the differences among the studied somaclones (somaclonal variation). The average over the two ratoon crops revealed that somaclones no. 7 and 8 surpassed the donor in highly significant values for most agronomic traits i.e. stalk height (14.35 and 9.48\%), stalk weight (9.52 and 15.24\%), stalk number/fed (21.00 and 31.25\%) and cane yield (32.16 and 52.02\%), respectively. Also, the somaclone no. 4 surpassed the donor in highly significant values for all technological traits i.e. sugar yield (23.52), brix (3.13), sucrose\% (6.28), purity\% (2.99), pol\% (6.36) and sugar recovery $\%(7.72 \%)$. The results concluded that the In-vitro cultures exhibited genetic variability among the obtained somaclones (somaclonal variation), which could be used to invent new superior somaclones and overcome the accomplishments of traditional cane breeding.
\end{abstract}

Keywords: Sugarcane; Immature leaves; Callus; Somaclones; Variation

\section{Introduction}

Sugarcane (Saccarum officinarum L.) is one of the most important crops in the world (Dagar et al., 2002). It is a clonally propagated from cuttings of stalk (Hoy et al., 2003), widely cultivated in the tropical to subtropical regions

\footnotetext{
*Corresponding author: A.B. El-Taib

Email; ashrafbakry71@yahoo.com

Received: January 20, 2021.

Accepted: March 3, 2021.

Published: March 22, 2021.
}

and annually provides around 60 to $70 \%$ of the world`s sugar (Shah et al., 2009). Recently, around $50 \%$ of the sugar production produced from sugarcane in Egypt. The local variety of GT-54 9 as a local commercial variety is grown since more than forty years. Also, the traditional breeding programs of sugarcane are slow, take long time, complicated efforts and costs to produce new varieties. Ho \& Vasil (1983) provided definitive evidence of the regeneration of plants from somatic embryos formed in tissue 
culture of sugarcane and the variation observed in the development of somatic embryos are neither unusual nor unexpected. Sugarcane tissue culture showed a wide range of genetic variation and rapid plant propagation (Murashige, 1974 and Rajeswari et al., 2009) and termed as somaclonal variation (Larkin \& Scowcroft, 1981). Somaclonal variation could prove to be a useful tool to overcome the difficulties in cane breeding (Bot et al., 2014). Moreover, subclonal variation (somaclonal variation) in sugarcane plays an important role in varietal improvement program (Krishnamurthi \& Tlaskal, 1974). Subclones or somaclones regenerated via tissue culture of sugarcane were superior to the donor genotypes in morphology traits, cane yield, weight of stalk, tillering, number of stalks, sucrose \% and quality traits (Sreenivasan \& Sreenivasan, 1984; Abo-Elwafa, 2011; Bot et al., 2014; Abo-Elwafa et al., 2015 and Swamy Gowada et al, 2016). High heritability and good genetic advance were observed for number of tillers and width of internode through sugarcane tissue culture (Solangi et al., 2016)). Moreover, Rastogi et al., (2015) detected that the somaclonal variations are easily achieved sugarcane. These variations play an important role in crop improvement program. Genetic variations are heritable in next generation and important for crop improvement.

Significant differences found among the sugarcane somaclones each other and their donor for yield of cane and sugar and their attributed traits in plant cane, first and second ratoon crops (Abo-Elwafa et al., 2015). Also, the changes of chromosome numbers ( $2 \mathrm{n}$ ) were reported among sugarcane somaclones each other and comparing to their donor (Liu \& Chen, 1976 and Sreenivasan \& Jalaja, 1982). In addition to, these changes were revealed via DNA polymorphism among sugarcane somaclones (El-Geddawy,
2008; Shahid et al, 2011 and Tiwari et al, 2011) The present study was aimed to determine the genetic variability among some somaclones of sugarcane (second and third ratoons crops) derived from callus of immature leaves of GT 54 9 sugarcane variety.

\section{Materials and Methods}

The current study was carried out at the Experimental Farm of Agronomy Department, Faculty of Agricultural, Assiut University, Egypt during two successive years i.e. 2018/19 (second ratoon crop) and 2019/20 (third ratoon crop).

I- Genetic materials: The genetic materials of the current investigation were eleven somaclones (i.e. 1 11) and their donor GT-54 9; the commercial variety in Egypt. These somaclones were derived from the callus of immature leaves of the donor variety GT-54 9 at the Tissue Culture Laboratory of Agronomy Department, Faculty of Agricultural, Assiut University.

\section{II- Field procedures:}

The eleven somaclones and their donor GT-54 9 were sown on March $5^{\text {th }}, 2018$ in randomized complete block design (RCBD) of three replications. The plot size was $42 \mathrm{~m}^{2}$ which consisted of 10 rows, $5.25 \mathrm{~m}$ long and $80 \mathrm{~cm}$ apart. A total of 66 buds were determined in the two middle rows to calculate cane yield. The millable stalks of one randomized stool/plot were harvested for different analyses. Each sample should be at least $25 \mathrm{Kg}$ from each plot was taken at random, stripped, cleaned and squeezed by an electric pillot mill and used for quality traits. Also, the samples of 2 liters/plot were undertaken to measure quality traits. Moreover, the following traits were measured in each plot /replicate: 
1- Stalk height (SH), cm; was measured from soil surface to the top visible dewlap of 10 millable stalks.

2- Stalk dimeter (SD), cm; measured from the middle internodes of 10 millable stalks.

3- Stalk weight (SW), kg; was calculated on plot basis = Weight of millable cane yield / Number of millable cane.

4- Stalk number/fed (SNF); was calculated on a plot basis.

5- Cane yield (CY), ton/fed; was calculated on a plot basis.

6- Sugar yield (SY), ton/fed; was estimated by multiplying net cane yield (ton /fed) by sugar recovery $\%$.

7- Juice of 30 stalk sample from each plot was analyzed for determining the following traits:

8- Brix (BR); determined with a hydrometer.

9- Sucrose \% (SUS), kg: SUS of clarified juice was determined by using automated sacharimeter according to A.O.A.C. (1980).

10 - Purity \% (PU), was determined as SUS/BR*100.

11- Pol \% (PO), was calculated according to the following formula described by Meade \& Chen (1977).

12-Sugar recovery (SR), was calculated according to the formula described by Yadav \& Sharma, (1980).

\section{III- Statistical analysis}

The separate analysis of variance for the obtained data of each year as well as the combined analysis of both years were done as outlined by Steel $\boldsymbol{\&}$ Torrie, 1980. Moreover, the following analyses were done as:

1- The genotypic $\left(\sigma_{\mathrm{g}}^{2}\right)$ and phenotypic $\left(\sigma_{\mathrm{p}}^{2}\right)$ variances, were calculated according to AlJibouri et al. (1958).
2- The phenotypic and genotypic coefficients of variation, were estimated using the formula developed by Burton (1952).

3- Heritability in broad sense "H", was estimated as the ratio of genotypic $\left(\sigma_{g}^{2}\right)$ to the phenotypic $\left(\sigma_{p}^{2}\right)$ variance according to Walker (1960).

\section{Results and Discussion}

Significant or highly significant differences were obtained among the somaclones each other and their donor for all studied traits in second and third ratoon crops $(2018 / 19 \& 2019 / 20)$ (Table 1) and their combined analysis (Table 2). These results revealed that abundance genetic differences were found among those sugarcane somaclones each other and comparing to their donor. Moreover, the values of genotypic (GCV) and phenotypic coefficient of variations (PCV) for agronomic traits were ranged from $2.24 \&$ 3.16 for stalk diameter to $18.11 \& 18.53 \%$ for cane yield over both ratoon crops (Table 3 ). Also, GCV and PCV for technological traits varied from $0.68 \& 0.79$ for purity $\%$ to $17.65 \& 17.76 \%$ for sugar yield over both ratoon crops (Table 3 ). It is remark results that the cane and sugar yields exerted the highest values of GCV and PCV and vice versa for stalk diameter and purity $\%$ in the two categories of traits in both ratoon crops. The heritability in broad sense which accounted from the expected main square ranged from 50.39 (stalk diameter) to $95.53 \%$ (cane yield) in agronomic traits and from 73.02 (purity \%) to $98.78 \%$ (sugar yield) over the both ratoon crops (Table 3). The obtained significant differences coupled with GCV, PCV and heritability estimates explained the genetic differences among the studied somaclones regenerated from the immature leaves of GT- 549 variety of sugarcane which named somaclonal variation. 
Table 1: Mean square of studied traits in 2018/19 \& 2019/20 seasons.

\begin{tabular}{|c|c|c|c|c|c|c|c|c|c|c|c|}
\hline \multirow[b]{2}{*}{ S.O.V. } & \multirow[b]{2}{*}{ d.f. } & \multicolumn{2}{|c|}{ Stalk Height, cm. } & \multicolumn{2}{|c|}{ Stalk Diameter, cm. } & \multicolumn{2}{|c|}{ Stalk weight, kg } & \multicolumn{2}{|c|}{ Stalk number/fed } & \multicolumn{2}{|c|}{ Cane yield, ton/fed. } \\
\hline & & $2018 / 19$ & $2019 / 20$ & $2018 / 19$ & $2019 / 20$ & $2018 / 19$ & $2019 / 20$ & $2018 / 19$ & $2019 / 20$ & $2018 / 19$ & $2019 / 20$ \\
\hline Reps & 2 & 34.750 & 17.361 & 0.003 & 0.005 & 0.001 & $9.3 \mathrm{E}-05$ & 3430000 & 7680000 & 2.417 & 12.316 \\
\hline Geno. & 11 & $3051.091 * *$ & $2845.20 * *$ & $0.033 * *$ & $0.062 *$ & $0.052 * *$ & $0.034 * *$ & $1.16 \mathrm{E}+08 * *$ & $1.6 \mathrm{E}+08 * *$ & $233.652 * *$ & $238.425 * *$ \\
\hline Error & 22 & 16.568 & 25.694 & 0.007 & 0.028 & 0.003 & 0.0008 & 6102727 & 872727 & 4.964 & 3.274 \\
\hline
\end{tabular}

Table 1: count.

\begin{tabular}{|c|c|c|c|c|c|c|c|c|c|c|c|c|c|}
\hline \multirow[b]{2}{*}{ S.O.V. } & \multirow[b]{2}{*}{ d.f. } & \multicolumn{2}{|c|}{ Sugar yield, ton/fed. } & \multicolumn{2}{|c|}{ Brix \% } & \multicolumn{2}{|c|}{ Sucrose $\%$} & \multicolumn{2}{|c|}{ Purity $\%$} & \multicolumn{2}{|c|}{ Pol \% } & \multicolumn{2}{|c|}{ Sugar rec. $\%$} \\
\hline & & $2018 / 19$ & $2019 / 20$ & $2018 / 19$ & $2019 / 20$ & $2018 / 19$ & $2019 / 20$ & $2018 / 19$ & $2019 / 20$ & $2018 / 19$ & $2019 / 20$ & $2018 / 19$ & $2019 / 20$ \\
\hline Reps & 2 & 0.153 & 0.289 & 0.120 & 0.042 & 0.167 & 0.060 & 0.185 & 1.018 & 0.120 & 0.035 & 0.093 & 0.052 \\
\hline Geno. & 11 & $4.106 * *$ & $4.428 * *$ & 0.398* & $0.334 * *$ & $0.524 *$ & $1.282 * *$ & $1.758 * *$ & $7.407 * *$ & $0.342 *$ & $0.957 * *$ & $0.327 * *$ & $0.961 * *$ \\
\hline Error & 22 & 0.119 & 0.071 & 0.181 & 0.029 & 0.184 & 0.091 & 0.313 & 1.268 & 0.129 & 0.064 & 0.102 & 0.062 \\
\hline
\end{tabular}

Table 2: Combined analysis for all studied traits.

\begin{tabular}{|c|c|c|c|c|c|c|c|c|c|c|c|c|}
\hline S.O.V. & d.f. & $\begin{array}{c}\text { Stalk } \\
\text { Height, } \\
\text { cm. }\end{array}$ & $\begin{array}{c}\text { Stalk } \\
\text { Diameter, } \\
\mathrm{cm} .\end{array}$ & $\begin{array}{c}\text { Stalk } \\
\text { weight } \\
\text { kg }\end{array}$ & $\begin{array}{c}\text { Stalk } \\
\text { number/fed }\end{array}$ & $\begin{array}{l}\text { Cane } \\
\text { yield, } \\
\text { ton/fed. }\end{array}$ & $\begin{array}{l}\text { Sugar } \\
\text { yield, } \\
\text { ton/fed. }\end{array}$ & Brix \% & $\begin{array}{c}\text { Sucrose } \\
\%\end{array}$ & Purity $\%$ & Pol \% & $\begin{array}{l}\text { Sugar } \\
\text { rec.\% }\end{array}$ \\
\hline Years (Y) & 1 & $2156.05 * *$ & $0.94 * *$ & $0.23 * *$ & $5.68 \mathrm{E}+08 * *$ & 5.28 & $5.16 \mathrm{E}-05$ & $56.87 * *$ & $8.03 * *$ & $265.74 * *$ & $2.37 * *$ & 0.42 \\
\hline $\begin{array}{c}\text { Reps. With. } \\
\text { year }\end{array}$ & 4 & $26.06 * *$ & 0.004 & 0.001 & 5555000 & 7.37 & 0.22 & 0.08 & 0.11 & 0.60 & 0.08 & 0.07 \\
\hline Genotypes (G) & 11 & $3958.75 * *$ & $0.06^{*}$ & $0.08 * *$ & $2.47 \mathrm{E}+08 * *$ & $451.86 * *$ & $8.12 * *$ & $0.58 * *$ & $1.41 * *$ & $5.65 * *$ & $1.01 * *$ & $0.98 * *$ \\
\hline $\mathrm{G}^{*} \mathrm{Y}$ & 11 & $1937.54 * *$ & 0.04 & $0.01 * *$ & $24583182 * *$ & $20.22 * *$ & $0.41 * *$ & 0.16 & $0.40 * *$ & $3.51 * *$ & $0.29 * *$ & $0.31 * *$ \\
\hline Pooled error & 44 & 21.13 & 0.02 & 0.002 & 3487727 & 4.12 & 0.10 & 0.11 & 0.14 & 0.79 & 0.10 & 0.08 \\
\hline
\end{tabular}


Abo-Elwafa et al., : SVU-International Journal of Agricultural Sciences,3 (1): 129-139, 2021

Table 3: Means of studied traits in second, third (2018/19, 2019/20) and over the two ratoons crops.

\begin{tabular}{|c|c|c|c|c|c|c|c|c|c|c|c|c|c|c|c|}
\hline \multirow{2}{*}{ Genotypes } & \multicolumn{3}{|c|}{ Stalk Height, cm. } & \multicolumn{3}{|c|}{ Stalk Diameter, cm. } & \multicolumn{3}{|c|}{ Stalk weight, kg } & \multicolumn{3}{|c|}{ Stalk number/fed } & \multicolumn{3}{|c|}{ Cane yield, ton/fed. } \\
\hline & $2018 / 19$ & $2019 / 20$ & Average & $2018 / 19$ & $2019 / 20$ & Average & $2018 / 19$ & $2019 / 20$ & Average & $2018 / 19$ & $2019 / 20$ & Average & $2018 / 19$ & $2019 / 20$ & Average \\
\hline GT54-9 & 267.33 & 260 & 263.67 & 2.51 & 2.19 & 2.35 & 0.95 & 1.15 & 1.05 & 43400 & 36600 & 40000 & 41.34 & 42.23 & 41.79 \\
\hline 1 & 283.33 & 246.67 & 265 & 2.45 & 2.17 & 2.31 & 1.04 & 1.08 & 1.06 & 53200 & 48000 & 50600 & 55.29 & 51.79 & 53.54 \\
\hline 2 & 263.67 & 256.67 & 260.17 & 2.65 & 2.4 & 2.53 & 1.02 & 1.15 & 1.08 & 44800 & 35400 & 40100 & 45.5 & 40.73 & 43.11 \\
\hline 3 & 263.33 & 220 & 241.67 & 2.48 & 2.29 & 2.39 & 1.04 & 1.16 & 1.1 & 46200 & 39600 & 42900 & 48.14 & 45.98 & 47.06 \\
\hline 4 & 255.67 & 220 & 237.83 & 2.52 & 2.23 & 2.37 & 1.08 & 1.05 & 1.06 & 47600 & 42600 & 45100 & 51.36 & 44.75 & 48.05 \\
\hline 5 & 247.33 & 203.33 & 225.33 & 2.37 & 2.44 & 2.41 & 0.87 & 1.01 & 0.94 & 39200 & 33600 & 36400 & 33.98 & 33.84 & 33.91 \\
\hline 6 & 230 & 226.67 & 228.33 & 2.47 & 2.37 & 2.42 & 0.82 & 1.02 & 0.92 & 53200 & 43800 & 48500 & 43.37 & 44.5 & 43.93 \\
\hline 7 & 303 & 300 & 301.5 & 2.46 & 2.34 & 2.4 & 1.1 & 1.19 & 1.15 & 51800 & 45000 & 48400 & 56.68 & 53.77 & 55.23 \\
\hline 8 & 297.33 & 280 & 288.67 & 2.61 & 2.02 & 2.31 & 1.15 & 1.28 & 1.21 & 54600 & 50400 & 52500 & 62.72 & 64.35 & 63.53 \\
\hline 9 & 188.33 & 276.67 & 232.5 & 2.53 & 2.29 & 2.41 & 0.69 & 0.96 & 0.82 & 57400 & 46200 & 51800 & 39.4 & 44.24 & 41.82 \\
\hline 10 & 231.67 & 240 & 235.83 & 2.67 & 2.55 & 2.61 & 0.95 & 0.98 & 0.96 & 54600 & 58800 & 56700 & 51.91 & 57.54 & 54.72 \\
\hline 11 & 237 & 206.67 & 221.83 & 2.73 & 2.42 & 2.57 & 0.91 & 0.93 & 0.92 & 39200 & 37800 & 38500 & 35.67 & 35.15 & 35.41 \\
\hline Average & 255.67 & 244.72 & 250.19 & 2.54 & 2.31 & 2.42 & 0.97 & 1.08 & 1.02 & 48767 & 43150 & 45958 & 47.11 & 46.57 & 46.84 \\
\hline $\operatorname{LSD}_{0.05}$ & 6.89 & 8.58 & 5.35 & 0.14 & 0.28 & 0.15 & 0.1 & 0.05 & 0.05 & 4183 & 1582 & 2175 & 3.77 & 3.06 & 2.36 \\
\hline $\operatorname{LSD}_{0.01}$ & 9.37 & 11.67 & 7.15 & 0.19 & 0.38 & 0.21 & 0.14 & 0.07 & 0.07 & 5686 & 2150 & 2906 & 5.13 & 4.16 & 3.16 \\
\hline G.C.V. & 12.44 & 12.53 & 7.34 & 3.67 & 4.62 & 2.24 & 13.13 & 9.83 & 10.09 & 12.43 & 16.63 & 13.25 & 18.53 & 19.01 & 18.11 \\
\hline P.C.V. & 12.47 & 12.58 & 7.37 & 4.13 & 6.23 & 3.16 & 13.60 & 9.96 & 10.26 & 12.77 & 16.68 & 13.36 & 18.73 & 19.14 & 18.53 \\
\hline H. & 99.46 & 99.10 & 98.97 & 79.00 & 54.99 & 50.39 & 93.31 & 97.49 & 96.71 & 94.75 & 99.44 & 98.46 & 97.88 & 98.63 & 95.53 \\
\hline$\delta^{2} \mathrm{G}$ & 1011.51 & 939.84 & 336.87 & 0.01 & 0.01 & 0.00 & 0.02 & 0.01 & 0.01 & 36750000 & 51513636 & 37100000 & 76.23 & 78.38 & 71.94 \\
\hline$\delta^{2} \mathrm{P}$ & 1017.03 & 948.40 & 340.39 & 0.01 & 0.02 & 0.01 & 0.02 & 0.01 & 0.01 & 38784242 & 51804545 & 37681288 & 77.88 & 79.47 & 75.31 \\
\hline
\end{tabular}


Abo-Elwafa et al., : SVU-International Journal of Agricultural Sciences,3 (1): 129-139, 2021

Table 3: continue.

\begin{tabular}{|c|c|c|c|c|c|c|c|c|c|c|c|c|c|c|c|c|c|c|}
\hline \multirow{2}{*}{$\begin{array}{c}\text { Genotype } \\
\text { s }\end{array}$} & \multicolumn{3}{|c|}{ Sugar yield, ton/fed. } & \multicolumn{3}{|c|}{ Brix \% } & \multicolumn{3}{|c|}{ Sucrose $\%$} & \multicolumn{3}{|c|}{ Purity $\%$} & \multicolumn{3}{|c|}{ Pol $\%$} & \multicolumn{3}{|c|}{ Sugar Rec. \% } \\
\hline & $\begin{array}{c}2018 / \\
19\end{array}$ & $\begin{array}{c}2019 / \\
20\end{array}$ & $\begin{array}{c}\text { Averag } \\
\mathrm{e}\end{array}$ & $\begin{array}{c}2018 / \\
19\end{array}$ & $\begin{array}{c}2019 / \\
20\end{array}$ & $\begin{array}{c}\text { Averag } \\
\mathrm{e}\end{array}$ & $\begin{array}{c}2018 / \\
19\end{array}$ & $\begin{array}{c}2019 / \\
20\end{array}$ & $\begin{array}{c}\text { Averag } \\
\mathrm{e}\end{array}$ & $\begin{array}{c}2018 / \\
19\end{array}$ & $\begin{array}{c}2019 / \\
20\end{array}$ & $\begin{array}{c}\text { Averag } \\
\mathrm{e}\end{array}$ & $\begin{array}{c}2018 / \\
19\end{array}$ & $\begin{array}{c}2019 / \\
20\end{array}$ & $\begin{array}{c}\text { Averag } \\
\mathrm{e}\end{array}$ & $\begin{array}{c}2018 / 1 \\
9\end{array}$ & $\begin{array}{c}2019 / 2 \\
0\end{array}$ & $\begin{array}{c}\text { Averag } \\
\mathrm{e}\end{array}$ \\
\hline GT54-9 & 5.61 & 5.87 & 5.74 & 21.59 & 23.75 & 22.67 & 19.41 & 20.38 & 19.9 & 89.87 & 85.81 & 87.84 & 16.33 & 16.69 & 16.51 & 13.56 & 13.90 & 13.73 \\
\hline 1 & 7.29 & 7.05 & 7.17 & 21.25 & 23.52 & 22.39 & 18.98 & 20.04 & 19.51 & 89.29 & 85.19 & 87.24 & 15.82 & 16.42 & 16.12 & 13.19 & 13.61 & 13.4 \\
\hline 2 & 6.17 & 5.56 & 5.87 & 21.47 & 23.51 & 22.49 & 19.41 & 20.08 & 19.75 & 90.43 & 85.17 & 87.8 & 16.21 & 16.58 & 16.4 & 13.57 & 13.65 & 13.61 \\
\hline 3 & 6.82 & 6.52 & 6.67 & 22.33 & 23.65 & 22.99 & 20.24 & 20.64 & 20.44 & 90.62 & 87.25 & 88.94 & 16.87 & 17.01 & 16.94 & 14.16 & 14.18 & 14.17 \\
\hline 4 & 7.33 & 6.84 & 7.09 & 22.45 & 24.31 & 23.38 & 20.39 & 21.9 & 21.15 & 90.84 & 90.1 & 90.47 & 17 & 18.12 & 17.56 & 14.29 & 15.28 & 14.79 \\
\hline 5 & 4.76 & 4.57 & 4.66 & 21.93 & 23.42 & 22.68 & 19.96 & 19.9 & 19.93 & 91.03 & 86.64 & 88.84 & 16.64 & 16.5 & 16.57 & 14.00 & 13.50 & 13.75 \\
\hline 6 & 5.92 & 5.97 & 5.94 & 21.67 & 23.2 & 22.44 & 19.54 & 19.75 & 19.65 & 90.17 & 85.2 & 87.69 & 16.31 & 16.19 & 16.25 & 13.64 & 13.41 & 13.53 \\
\hline 7 & 7.68 & 7.19 & 7.43 & 21.74 & 23.14 & 22.44 & 19.47 & 19.69 & 19.58 & 89.56 & 85.09 & 87.32 & 16.25 & 16.15 & 16.2 & 13.55 & 13.37 & 13.46 \\
\hline 8 & 8.4 & 8.75 & 8.57 & 21.35 & 23.47 & 22.41 & 19.2 & 20.01 & 19.61 & 89.92 & 85.26 & 87.59 & 16.08 & 16.6 & 16.34 & 13.39 & 13.6 & 13.5 \\
\hline 9 & 5.3 & 6.34 & 5.82 & 22 & 23.91 & 22.96 & 19.45 & 20.94 & 20.19 & 88.38 & 87.53 & 87.96 & 16.24 & 17.37 & 16.81 & 13.45 & 14.33 & 13.89 \\
\hline 10 & 6.96 & 7.78 & 7.37 & 21.68 & 23.36 & 22.52 & 19.33 & 19.9 & 19.62 & 89.12 & 85.21 & 87.17 & 16.11 & 16.5 & 16.31 & 13.42 & 13.52 & 13.47 \\
\hline 11 & 4.83 & 4.66 & 4.75 & 21.65 & 23.21 & 22.43 & 19.45 & 19.62 & 19.54 & 89.86 & 84.54 & 87.2 & 16.19 & 16.28 & 16.24 & 13.56 & 13.27 & 13.42 \\
\hline Average & 6.42 & 6.43 & 6.42 & 21.76 & 23.54 & 22.65 & 19.57 & 20.24 & 19.91 & 89.92 & 86.08 & 88.01 & 16.34 & 16.70 & 16.52 & 13.65 & 13.80 & 13.73 \\
\hline $\operatorname{LSD}_{0.05}$ & 0.59 & 0.45 & 0.36 & 0.72 & 0.29 & 0.38 & 0.73 & 0.51 & 0.43 & 0.95 & 1.91 & 1.04 & 0.61 & 0.43 & 0.36 & 0.54 & 0.42 & 0.33 \\
\hline $\operatorname{LSD}_{0.01}$ & 0.8 & 0.61 & 0.48 & 0.98 & 0.39 & 0.5 & 0.99 & 0.7 & 0.58 & 1.29 & 2.59 & 1.38 & 0.83 & 0.58 & 0.48 & 0.74 & 0.57 & 0.45 \\
\hline G.C.V. & 17.95 & 18.76 & 17.65 & 1.24 & 1.35 & 1.17 & 1.72 & 3.11 & 2.06 & 0.77 & 1.66 & 0.68 & 1.63 & 3.27 & 2.10 & 2.01 & 3.96 & 2.43 \\
\hline P.C.V. & 18.22 & 18.91 & 17.76 & 1.67 & 1.42 & 1.30 & 2.14 & 3.23 & 2.19 & 0.85 & 1.83 & 0.79 & 2.07 & 3.38 & 2.24 & 2.42 & 4.10 & 2.58 \\
\hline $\mathrm{H}$. & 97.09 & 98.41 & 98.78 & 54.57 & 91.37 & 79.96 & 64.94 & 92.88 & 87.98 & 82.22 & 82.88 & 73.02 & 62.45 & 93.37 & 88.31 & 68.82 & 93.52 & 89.08 \\
\hline$\delta^{2} \mathrm{G}$ & 1.33 & 1.45 & 1.29 & 0.07 & 0.10 & 0.07 & 0.11 & 0.40 & 0.17 & 0.48 & 2.05 & 0.36 & 0.07 & 0.30 & 0.12 & 0.08 & 0.30 & 0.11 \\
\hline$\delta^{2} \mathrm{P}$ & 1.37 & 1.48 & 1.30 & 0.13 & 0.11 & 0.09 & 0.17 & 0.43 & 0.19 & 0.59 & 2.47 & 0.49 & 0.11 & 0.32 & 0.14 & 0.11 & 0.32 & 0.13 \\
\hline
\end{tabular}


The genetic variation among plantlets of sugarcane produced by tissue cultures was reported by many authors such as Sreenivasan \& Jalaja (1982), Sreenivasan \& Sreenivasan (1984), Abo-Elwafa (2011), Sobhakumari (2012), AboElwafa et al., (2015) and Gadakh, et al. (2015). Also, the somaclonal variation in sugarcane was reported by Abo-Elwafa (2004), Wagih, et al. (2004), Doule, et al. (2008), Roy, et al. (2010), Shomeili, et al. (2011) and Dalvi, et al. (2012).

The data in Tables 3 revealed that all agronomic traits, except stalk weight, possessed high values in second ratoon (2018/19) comparing to third ratoon crop (2019/20) relative to the general means of all genotypes. These values were 4.47, 9.96, 13.02 and $1.22 \%$ for stalk height, stalk diameter, stalk number/fed and cane yield, respectively. This view was vice versa for all technological traits, except purity \%, which their values were high in third ratoon (2019/20) comparing to the second ratoon crop (2018/19) and accounted $0.20,8.18,3.42,2.20$ and $1.10 \%$ for sugar yield, brix $\%$, sucrose $\%$, pol $\%$ and sugar recovery, respectively, relative to the general means of all genotypes.

It is remarkable results on the base of average over the two ratoon crops that somaclones no. 7 and 8 surpassed the donor in highly significant values for most agronomic traits i.e. stalk height (14.35 and $9.48 \%)$, stalk weight (9.52 and $15.24 \%$ ), stalk number/fed (21.00 and 31.25\%) and cane yield (32.16 and $52.02 \%$ ), respectively. Also, the somaclone no. 4 surpassed the donor in highly significant values for all technological traits i.e. sugar yield (23.52\%), brix (3.13\%), sucrose\% (6.28\%), purity\% (2.99\%), pol\% (6.36\%) and sugar recovery\% (7.72\%) (Tables $3 \& 4)$.

Moreover, there were other somaclones surpassed the donor in significant or highly significant values for the agronomic traits i.e. no. $2(7.66 \%)$, no. $10(11.06 \%)$ and no. $11(9.36 \%)$ for stalk diameter; no. 3 (4.76\%) for stalk weight; no. $1(26.50 \%)$, no. $3(7.25 \%)$, no. $4(12.75 \%)$, no. $6(21.25 \%)$, no. $9(29.50 \%)$ and no. 10 $(41.75 \%)$ for stalk number/fed; and no. 1 (28.12\%), no. 3 (12.61\%), no. $4(14.98 \%)$ and no. $10(30.94 \%)$ for cane yield. Also, there were other somaclones exceeded the donor in significant or highly significant values for the technological traits i.e. no. 1 (24.91\%), no. 3 (16.20\%), no. 7 (29.44\%), no. $8(49.30 \%)$ and no. $10(28.40 \%)$ for sugar yield; no. $3(2.21 \%)$ for sucrose $\%$; no. 3 (1.25\%) for purity; no. $3(2.60 \%)$ for pol\%; and no. 3 (3.20\%) for sugar recovery $\%$. (Tables 3 \& 4).

In addition to, the best and highest somaclones exceeded their donor on the average over the two ratoon crops were no. 7 for stalk height (14.35\%); no. 10 for stalk diameter $(11.06 \%)$ and stalk number/fed (41.75\%); no. 8 for stalk weight $(15.24 \%)$, cane yield $(52.02 \%)$ and sugar yield (49.30\%); and no. 4 for brix (3.13\%), sucrose\% (6.28\%), purity\% (2.99\%), pol\% $(6.36 \%)$ and sugar recovery\% $(7.72 \%)$ (Tables $3 \& 4$ ). Some somaclones were possessed significant or highly significant positive realized increasing values comparing to their donor in one ratoon crop (second or third) such as in second ratoon crop no. 1 for stalk height (5.99\%), no. 2 for cane yield (10.06\%), no. 3 for brix (3.43\%) and no. 5 for purity $(1.29 \%)$; and in third ratoon crop no. 9 for stalk height (6.41\%), sugar yield (8.01\%), sucrose\% (2.75\%), pol\% (4.07) and sugar recovery\% (3.09) (Tables $3 \& 4$ ).

The obtained results concluded that the In-vitro cultures through immature leaves of the donor exhibited genetic variability in all studied traits among the obtained somaclones which regenerated from the same donor, supporting the 
Table 4: Direct response of the somaclones comparative to the donor GT54-9 for studied traits in 2018/19, 2019/20 and over both ratoons crop.

\begin{tabular}{|c|c|c|c|c|c|c|c|c|c|c|c|c|c|c|c|}
\hline \multirow{2}{*}{ Genotypes } & \multicolumn{3}{|c|}{ Stalk Height, cm. } & \multicolumn{3}{|c|}{ Stalk Diameter, cm. } & \multicolumn{3}{|c|}{ Stalk weight, $\mathrm{kg}$} & \multicolumn{3}{|c|}{ Stalk number/fed } & \multicolumn{3}{|c|}{ Cane yield, ton/fed. } \\
\hline & $2018 / 19$ & $2019 / 20$ & Average & $2018 / 19$ & $2019 / 20$ & Average & $2018 / 19$ & $2019 / 20$ & Average & $2018 / 19$ & $2019 / 20$ & Average & $2018 / 19$ & $2019 / 20$ & Average \\
\hline 1 & 5.99 & -5.13 & 0.50 & -2.39 & -0.91 & -1.70 & 9.47 & -6.09 & 0.95 & 22.58 & 31.15 & 26.50 & 33.74 & 22.64 & 28.12 \\
\hline 2 & -1.37 & -1.28 & -1.33 & 5.58 & 9.59 & 7.66 & 7.37 & 0.00 & 2.86 & 3.23 & -3.28 & 0.25 & 10.06 & -3.55 & 3.16 \\
\hline 3 & -1.50 & -15.38 & -8.34 & -1.20 & 4.57 & 1.70 & 9.47 & 0.87 & 4.76 & 6.45 & 8.20 & 7.25 & 16.45 & 8.88 & 12.61 \\
\hline 4 & -4.36 & -15.38 & -9.80 & 0.40 & 1.83 & 0.85 & 13.68 & -8.70 & 0.95 & 9.68 & 16.39 & 12.75 & 24.24 & 5.97 & 14.98 \\
\hline 5 & -7.48 & -21.80 & -14.54 & -5.58 & 11.42 & 2.55 & -8.42 & -12.17 & -10.48 & -9.68 & -8.20 & -9.00 & -17.80 & -19.87 & -18.86 \\
\hline 6 & -13.96 & -12.82 & -13.40 & -1.59 & 8.22 & 2.98 & -13.68 & -11.30 & -12.38 & 22.58 & 19.67 & 21.25 & 4.91 & 5.38 & 5.12 \\
\hline 7 & 13.34 & 15.38 & 14.35 & -1.99 & 6.85 & 2.13 & 15.79 & 3.48 & 9.52 & 19.35 & 22.95 & 21.00 & 37.11 & 27.33 & 32.16 \\
\hline 8 & 11.22 & 7.69 & 9.48 & 3.98 & -7.76 & -1.70 & 21.05 & 11.30 & 15.24 & 25.81 & 37.70 & 31.25 & 51.72 & 52.38 & 52.02 \\
\hline 9 & -29.55 & 6.41 & -11.82 & 0.80 & 4.57 & 2.55 & -27.37 & -16.52 & -21.90 & 32.26 & 26.23 & 29.50 & -4.69 & 4.76 & 0.07 \\
\hline 10 & -13.34 & -7.69 & -10.56 & 6.37 & 16.44 & 11.06 & 0.00 & -14.78 & -8.57 & 25.81 & 60.66 & 41.75 & 25.57 & 36.25 & 30.94 \\
\hline 11 & -11.35 & -20.51 & -15.87 & 8.76 & 10.50 & 9.36 & -4.21 & -19.13 & -12.38 & -9.68 & 3.28 & -3.75 & -13.72 & -16.77 & -15.27 \\
\hline Average & -4.76 & -6.41 & -5.58 & 1.20 & 5.94 & 3.40 & 2.11 & -6.64 & -2.86 & 13.49 & 19.52 & 16.25 & 15.24 & 11.22 & 13.19 \\
\hline
\end{tabular}

Table 4: Count.

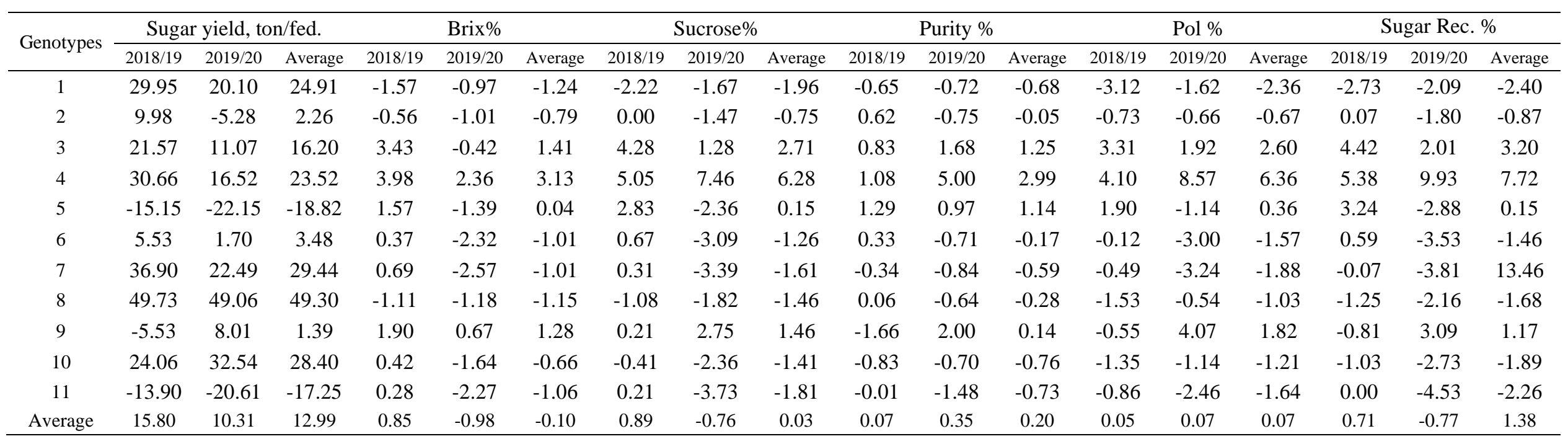


effective current technique to obtain somaclonal variation which could be used in breeding and selection of sugarcane to invent new somaclones genetically different and overcome the difficulties and accomplishments in traditional cane breeding. Same results were found by AboElwafa (2004), Rajeswari, et al., (2009), AboElwafa (2011), Abo-Elwafa et al., (2015), Sobhakumari (2012), Bot et al., (2014) and Gadakh et al., (2015), Biradar et al., (2016), Rina et al., (2017), Solangi et al., (2016), Tripathy et al., (2016) and Swamy Gowda et al., (2016).

The present study suggests that the obtained somaclones through in vitro cultures can be exploited to develop and improve the agronomical and technological traits in short time comparing to conventional breeding programs in sugarcane.

\section{References}

Abo-Elwafa, A. (2004) 'In field assessment of somaclonal variability in Sugarcane through callus culture' Assiut. J. of Agric. Soi., 35(4): 29-47.

Abo-Elwafa, A. (2011) 'In field assessment of somaclonal variation among sugarcane clones derived through immature leaf callus culture' Egyptian Sugar J. 4: 1-19.

Abo-Elwafa, A., Hamada, A., Nosaer, H. and Faheim, H. (2015) 'Assessment of somaclonal variation, correlation and stepwise regression to evaluate new sugarcane somaclones' Egyptian Sugar J. 8: 131-150.

Al-Jibouri, H.A., Miller, P.A. and Robinson, H.F. (1958) 'Genotypic and environmental variation and covariance in an Upland cotton cross of interspecific origin' Agron. J. 50: 633-636.
A.O.A.C. (1980) 'Official Methods of analysis' Association of official Analytical Chemists, Washington, D.C., USA.

Biradar, V., Patil, M.S., Mashetty, S.B., Lachuriye, P. and Shrigiri, P. (2016) 'Regeneration of somaclones from sugarcane cultivar co-740 \& comparison of their agronomic characters with local varieties' Int. J. Adv. Res., 4(10): 397-406.

Bot, P.J., Raza, S., Qamarunnisa, S., Jamil, I., Naqvi, B., Azhar, A. and Quresh, J.A. (2014) 'Screening of sugarcane somaclones of variety BL4 for agronomic characteristics' Pakistan J. of Botany 45(4): 1532-11535.

Burton, G.W. (1952) 'Quantitative inheritance in grasses'6th Int. Grassland Cong. Proc., 1: 227-283.

Dagar, P., Pahja, S.K., Kadian, S.P. and Singh, S. (2002) 'Evaluation of phenotypic varability in sugarcane using principal factor analysis' Indian J. of sugarcane Tech. 17: 95-100.

Dalvi, S.G., Vasekar, V.C., Yadav, A., Tawar, P.N., Dixit, G.B., Theerthaprasad D. and Deshmukh, R. B. (2012) 'Screening of promising Sugarcane Somaclones for agronomic Traits and Smut Resistance Using PCR Amplification of Inter Transcribed Region (ITS) of Sprisorium Scitamineae' Sugar Tech., 14 (1):68-75.

Doule, R.B., Kawar, P.G., Devarumath, R.M. and Nerkar, Y.S. (2008) 'Field performance and RAPD analysis for assessment of genetic variation in sugarcane somaclones' Indian Journal of Genetics and Plant Breeding, 68(3):301- 306 .

El-Geddawy, D.H., Azzam, C. and Khalil, S. M. (2008) 'Somaclonal variation in sugarcane through tissue culture and subsequent screening for molecular polymorphisms' Egypt. J. Genet. Cytol., 37: 335-358. 
Gadakh, S.S., Patel, D.U. and Patil, A.B. (2015) 'Evaluation of sugarcane (Saccharum spp. Ccomlex) Mutants for yield Contributing Traits and Quality Parameters" I.J.A.B.R., 5(3): 220-228.

Ho, W. and Vasil, I.K. (1983) "Somatic embrogenesis in sugarcane (Saccharum officinarum L.) I. The morphology and physiology of callus formation and the ontogeny of somatic embryos' Protoplasma, 118: 169-180.

Hoy, J.W., Bischoff, K.P. Milligan, S.B. and Gravois, K.A. (2003) 'Effect of tissue culture explant source on sugarcane yield components' Euphytica, 129(2): 237-240.

Krishnamurthi, M. and Tiaskal, J. (1974) 'Fiji disease resistant Saccharum officinarum var Pindar subclones from tissue culture' Proc. Tnt. Soc. Sugarcane Technol., 15 (1): 130137.

Larkin, P.J. and Scowcroft, W.R. (1981) 'Somaclonal Variation - a Novel Source of Variability from Cell Cultures for Plant improvement' Theor. Appl. Genet, 60(4):197-214.

Liu, M. and Chen, W. (1976) 'Tissue and Cell Culture as Aids to Sugarcane Breeding. I. Creation of Genetic Variation Through Callus Culture' Euphytica, 25:393-403.

Meade, G.P. and Chen, J.C.P. (1977) 'Cane Sugar Hand Book' $10^{\text {th }}$ Ed. A Willy Inter. Science Publication, John Wiley \& Sons, New York, London, Sydney.

Murashige, T. (1974) 'Plant propagation through tissue cultures' A. Rev. Plant Physiol. 25: 135-166.

Rajeswari S, S. Thirugnanakumar, A. Anandan and M. Krishnamurthi. 2009. 'Somaclonal variation in sugarcane through tissue culture and evaluation for quantitative and quality traits' Euphytica, 168: 7 1-80.
Rastogi, J. Siddhant., Bubber, P. and Sharma, B.L. (2015) 'Somaclonal Variation: A new dimension for sugarcane improvement' Gerf Bulletin Biosciences, 6(1):5-10 .

Rina, P.D., Mali, S.C., Narwade, A.V., Patel, A.I. and Udhutha, J. (2017) 'Effect of different concentrations of 2, 4-dichlorophenoxy acetic acid on callus induction of sugarcane (Saccharum officinarum L.) Var. Co 86032 and com 0265' International Journal of Chemical Studies, 5 (4): 38-40.

Roy, M., Hossain, M., Biswas, A., Islam, R., Sarker, S.R. and Akhter, S. (2010) 'Induction and Evaluation of Somaclonal Variation in Sugarcane (Saccharum officinarum L.) var. Isd-16. Gene Conserve, 9 (38): 201-221.

Shah, A.H., Rashid, N., Haider, M.S., Saleem, F., Tahir, M. and Iqbal, J. (2009) 'An efficient short and cost effective regeneration system for transformation studies of sugarcane (Saccharum officinarum L.) Pak' J. Bot., 42 (2): 609-614.

Shahid, M.T., Khan, F.A., Saeed, A. and Fareed, I. (2011) 'Variability of red rot-resistant somaclones of sugarcane genotype s97us297 assessed by RAPD and SSR' Genet Mol. Res., 10(3): 1831-1849.

Shomeili, M., Majid, N., Mosa, M. and Rajabi, M. (2011) 'Evaluation of sugarcane (Saccharum officinarum L.) somaclonal variants tolerance to salinity in vitro and in vivo cultures' African Journal of Biotechnology, 10(46)

Sobhakumari, V.P. (2012) "Assessment of somaclonal variation in sugarcane" African Journal of Biotechnology, 11 (87): 1530315309.

Solangi, S. K., Qureshi, S.T., Solangi, N., Solangi, M.K., Khan, I.A., Parveen, A., Channar, A.P. and Hussain, A. (2016) "Heritability analysis to screen elite 
sugarcane (Saccharum spp.) soma clones under field conditions Solangi' Journal of Plant Breeding and Crop 8(9):168-174.

Sreenivasan, T.V. and Jalaja, N.C. (1982) 'Sugarcane varital improvement through tissues culture' In: Cell and culture in crop improvement, pp. 371-376. (eds S.K. Sen and K. Giles), New York, Plenum.

Sreenivasan, J. and Sreenivasan, T.V. (1984) 'In vitro propagation of a Saccharum officinarum (L.) and Sclerostachya fusca (Roxb.) A' Camus hybrid. Theor. Appl. Genet., 67(2\&3):171-174 .

Steel, R.G.D. and Torrie, J.H.. (1980) 'Principle and Procedures of Statistics' A Biometrical Approach 2nd Ed., McGraw-Hill Book Company, New York, U.S.A.

Swamy Gowda, S.N., Saravanan, K. and Ravishankar, C.R. (2016) 'Correlation and path analysis for yield and quality attributes in sugarcane' International $J$. of Sci. Technology \& Engineering, 3 (2), ISSN (online): 2349-784X.

Tiwari, A.K., Mishra, N., Tripathi, S., Lal, M., Singh, R.K. and sharma, M.L. (2011) 'Assessment of Genetic Stability in Micro Propagated Population of Sugarcane Variety CoS 07250 Through SSR Markers' International Journal of Plant Research, 24(2):75-81.

Tripathy, S.K., Panda, A., Pramod K., Nayak, Sasmita Dash, Lenka, D.D., Mishra, R., Rajesh, K., Kar, N. and Senapati, Dash, G.B. (2016) 'Somaclonal Variation for Genetic Improvement in Grasspea (Lathyrus sativus L.) ' Legume Research, 39 (3): 329335.

Wagih, M.E., Ala, A. and Musa, Y. (2004) 'Regeneration and evaluation of sugarcane somaclonal variants for drought tolerance' Sugar Tech., 6 (1): 35-40.
Walker, T.T. (1960) 'The use of a selection index technique in the analysis of progeny row data' Emp. Cott. Gr. Rev., 37: 81-107.

Yadav, R.L. and Sharma, R.K. (1980) 'Effect of nitrogen levels and harvesting dates on quality and yield of four sugar cane genotypes' Indian J., 50 (7): 581-589. 